\title{
Adaptation of the metabolomics profile of rice after Pyricularia oryzae infection
}

\begin{abstract}
Pyricularia oryzae (P. oryzae), one of the most devastating fungal pathogens, is the cause of blast disease in rice. Infection with a blast fungus induces biological responses in the host plant that lead to its survival through the termination or suppression of pathogen growth, and metabolite compounds play vital roles in plant interactions with a wide variety of other organisms. Numerous studies have indicated that rice has a multi-layered plant immune system that includes pre-developed (e.g., cell wall and phytoanticipins), constitutive and inducible (phytoalexins) defence barriers against stresses. Significant progress towards understanding the basis of the molecular mechanisms underlying the defence responses of rice to P. oryzae has been achieved. Nonetheless, even though the important metabolites in the responses of rice to pathogens have been identified, their exact mechanisms and their contributions to plant immunity against blast fungi have not been elucidated. The purpose of this review is to summarize and discuss recent advances towards the understanding of the integrated metabolite variations in rice after $P$. oryzae invasion.
\end{abstract}

Keyword: Fungal pathogen; Plant immunity system; Metabolite compounds; Blast disease; Phytoanticipins; Phytoalexins 